\title{
Non-invasive cardiac monitoring during caesarean delivery in a parturient with newly-diagnosed ventricular bigeminy
}

\author{
Wan Yen Lim ${ }^{1}$, Wan Ling Leong ${ }^{2}$, Rehena Sultana ${ }^{3}$ and Ban Leong Sng ${ }^{4}$ \\ ${ }^{1}$ Senior Resident, Singhealth Anaesthesiology Residency Program, Singapore \\ ${ }^{2}$ Consultant, Department of Women's Anaesthesia, KK Women's and Children's Hospital, Singapore \\ ${ }^{3}$ Associate Biostatistician, Duke-NUS Graduate Medical School, Singapore \\ ${ }^{4}$ Head and Senior Consultant, Department of Women's Anaesthesia, KK Women's and Children's Hospital, Singapore, Associate Professor, Duke-NUS Graduate \\ Medical School, Singapore
}

\section{Introduction}

Ventricular bigeminy is a ventricular arrhythmia that has the potential to degenerate into ventricular tachycardia or fibrillation. This is a case report highlighting the anaesthetic considerations of a parturient with newly-diagnosed ventricular bigeminy undergoing urgent Caesarean delivery. The use of intraoperative non-invasive cardiac monitoring and regional anaesthetic technique was employed to achieve stable maternal cardiac haemodynamics.

\section{Case description}

A 31 year old primigravida with no significant past medical history, was electively admitted for induction of labour at 38 weeks gestation. Maternal bradycardia was noted on monitoring and ECG performed revealed ventricular bigeminy. Heart rate ranged from 38-45 beats per minute. Cardiology referral revealed likely premature ventricular complexes (PVCs) possibly from right ventricular outflow tract obstruction. The options from the multidisciplinary consultation included (1) continuation with induction of labour with avoidance of labour oxytocin augmentation to prevent hypotension or (2) urgent Caesarean delivery. After further multidisciplinary discussion and informed consent, the decision was made for urgent Caesarean delivery. Pre-operative blood tests including full blood count, electrolytes, thyroid function tests and cardiac enzymes were normal.

Combined spinal-epidural (CSE) technique (Espocan, B Braun, Melsungen, Germany) was performed in the sitting position, at the L4-L5 interspace using the loss of resistance to saline to identify the epidural space. A spinal dose of $9 \mathrm{mg}$ of $0.5 \%$ hyperbaric bupivacaine, fentanyl $20 \mathrm{mcg}$ and morphine $100 \mathrm{mcg}$ was administered slowly. Loss of cold sensation was achieved at T4 level prior to surgical incision. Haemodynamic data (eg. heart rate, blood pressure, cardiac output) were monitored intraoperatively using non-invasive Nexfin cardiac monitor. Intermittent boluses of ephedrine (total $18 \mathrm{mg}$ ) were given to maintain haemodynamic stability during the episodes of hypotension associated with reduced cardiac output following the spinal dose. Figure 1 and Figure 2 shows the systolic pressure and cardiac output trends over time with intraoperative events marked. $1000 \mathrm{ml}$ of Ringer Lactate co-loading was administered.

A healthy $2.5 \mathrm{~kg}$ male baby with APGAR scores of 9 and 9 at 1 and 5 minutes was delivered. Intravenous carbetocin $100 \mathrm{mcg}$ as a slow bolus over 30 minutes was administered after delivery to reduce the risk of uterine atony. The patient was then transferred to the intensive care unit for haemodynamic monitoring. There were intermittent episodes of ventricular bigeminy, but she remained haemodynamically stable. Electrolytes remained within normal range. She was subsequently transferred to general ward the following day and discharged home on post-operative day 3 .

Cardiology consultation and transthoracic echocardiogram was performed 1 month later. The echocardiography assessment showed frequent PVCs occurring during ventricular bigeminy episodes. There was normal heart morphology with estimated ejection fraction of 50$55 \%$. She remained asymptomatic and subsequently a 24 hour Holter monitoring was performed, which revealed PVC load $>30 \%$ with highest grade being non-sustained ventricular tachycardia of 3 beats. Treadmill exercise testing conducted was negative for myocardial ischaemia. Bisoprolol (beta blocker) was commenced and ablation procedure was advised if cardiac symptoms developed.

\section{Discussion}

We report a case of ventricular bigeminy during pregnancy with close cardiac haemodynamic monitoring during CSE anaesthesia for Caesarean delivery. The non-invasive, continuous cardiac output monitor uses the finger arterial pulse-contour analysis. Arterial pressure is measured directly from a finger cuff using a volumeclamp method, where brachial arterial pressure is reconstructed using waveform filtering with pressure level correction [1]. Advantages include the beat-to-beat haemodynamic monitoring, ease of use and non-invasiveness. This could avoid the potential serious complications associated with invasive arterial line insertion, such as infection, haematoma, ischaemia and pseudoaneurysm.

The use of non-invasive cardiac monitoring may be useful in the perioperative period. There have been several reports of intraoperative ventricular bigeminy during orthopaedic procedures [2]. The use of non-invasive cardiac monitoring has been validated in the obstetric

Correspondence to: Ban Leong Sng, MBBS, MMed (Anaes), FANZCA, FFPMANZCA, MCI, FAMS, Deputy Head and Senior Consultant, Department of Women's Anaesthesia, KK Women's and Children's Hospital, Singapore, Associate Professor, Duke-NUS Graduate Medical School, 100 Bukit Timah Road, Singapore 229899, Tel: +65 63941081; E-mail: sng.ban.leong@singhealth.com.sg

Received: September 28, 2017; Accepted: October 20, 2017; Published: October 23,2017 


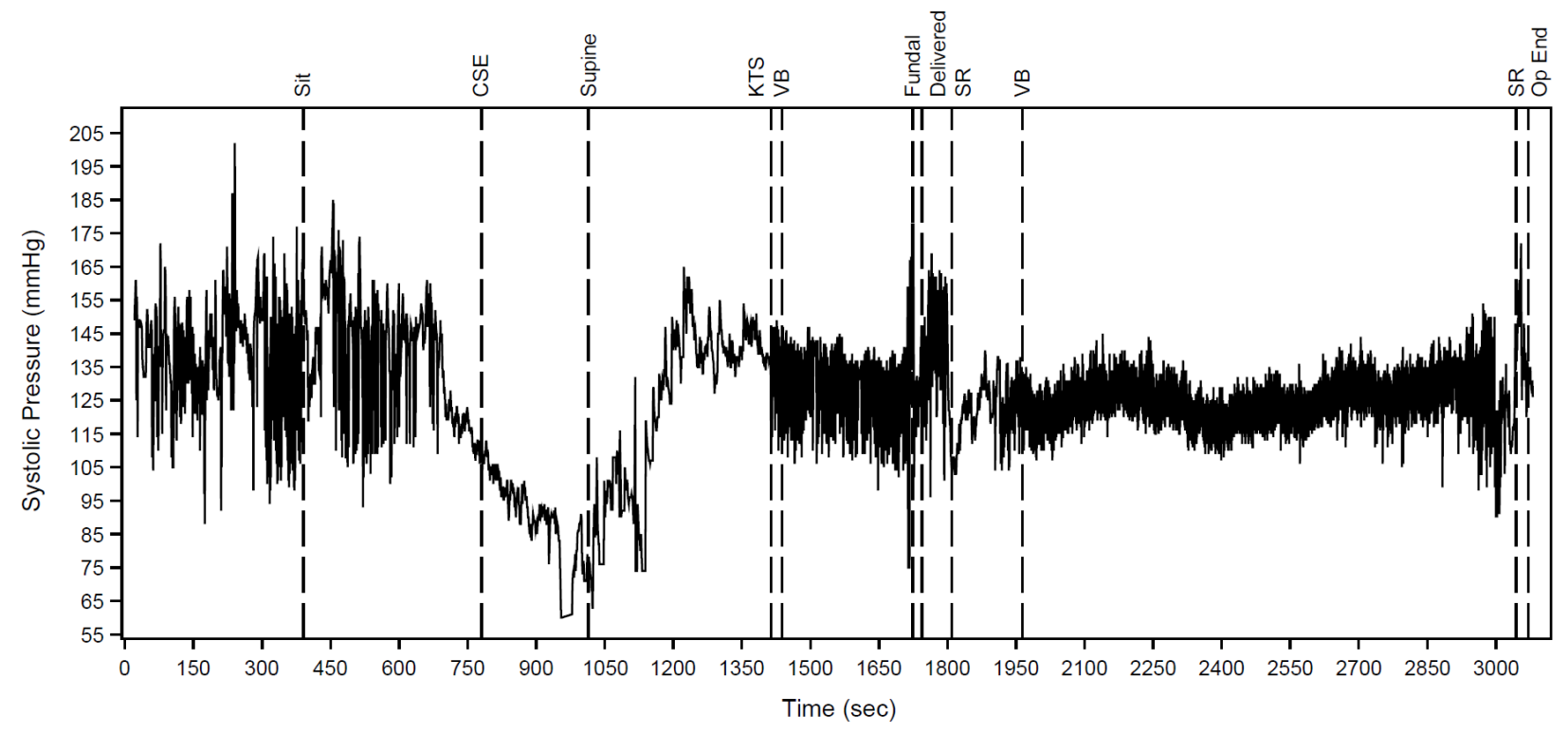

Sit: Sitting position

CSE: Combined spinal epidural

Supine: Supine position

KTS: Knife to skin

VB: ventricular bigeminy

Fundal: Fundal pressure

Delivered: Delivery of foetus

SR: Sinus rhythm

Op end: End of surgery

Figure 1. Graph of Systolic Blood Pressure versus Time during Caesarean delivery.

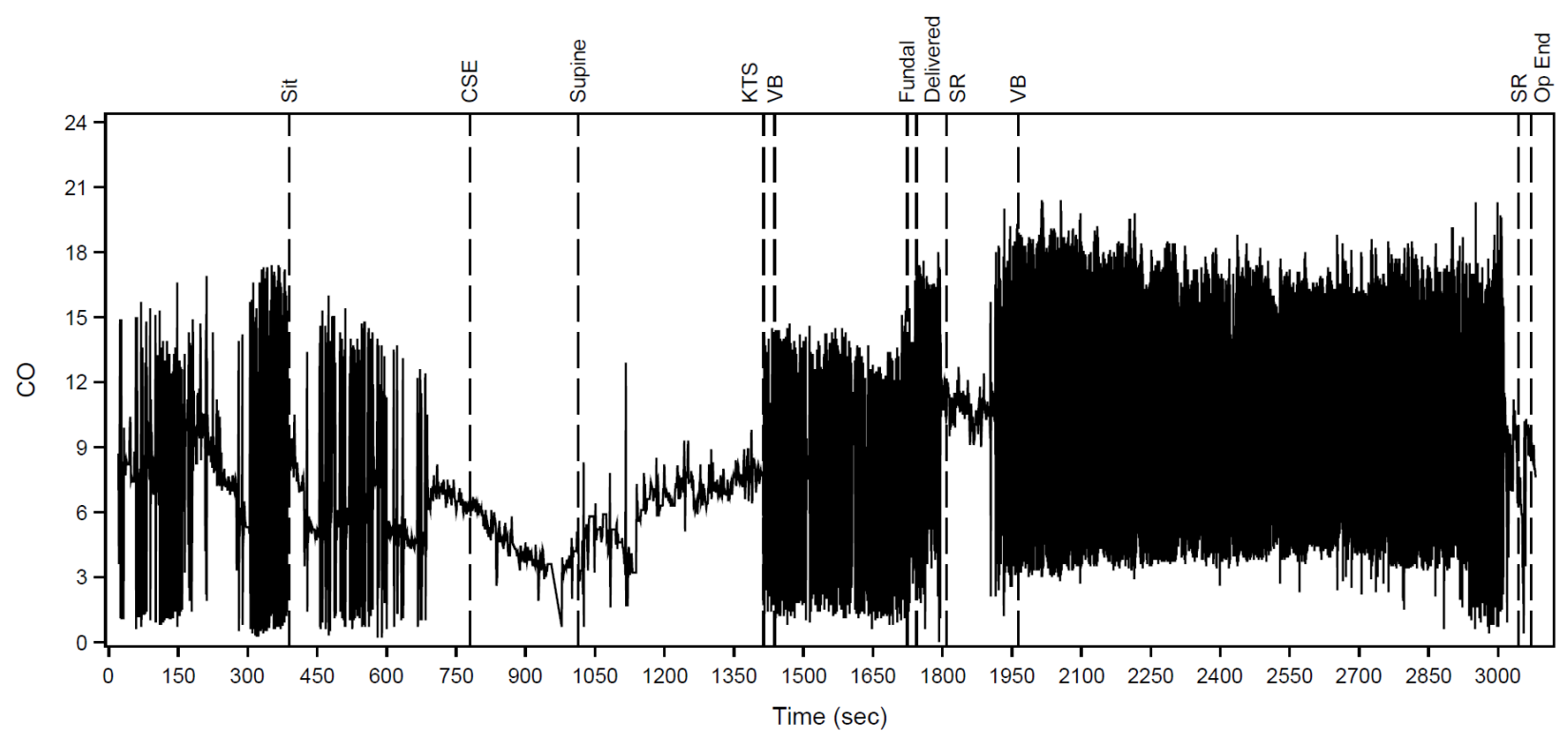

Figure 2. Graph of Cardiac Output versus time during Caesarean delivery.

Sit: Sitting position

CSE: Combined spinal epidural

Supine: Supine position

KTS: Knife to skin

VB: ventricular bigeminy

Fundal: Fundal pressure

Delivered: Delivery of foetus

SR: Sinus rhythm

Op end: End of surgery 
practice, where haemodynamic stability maintained during spinal anaesthesia for caesarean section led to good maternal and foetal outcomes [3]. A recent study also used cardiac monitoring to predict the risk of hypotension following spinal anaesthesia [4]. In the obstetric population where there is no autoregulation of utero-placental perfusion, maternal hypotension may lead to placental hypo-perfusion and possibly, foetal ischaemia.

The use of continuous non-invasive cardiac output and intraarterial blood pressure monitoring, could enable timely intervention using vasopressors to maintain haemodynamic stability. Good correlation was also established between cardiac output values obtained using Nexfin compared to invasive cardiac monitoring [5]. The limitation of non-invasive cardiac monitoring would be the relative large swings in values, although the trend of the monitoring parameters could still be useful. The presence of more than 5 PVCs per minute has been reported to increase cardiac risk in the perioperative period [6]. However, aggressive attempts to maintain sinus rhythm using amiodarone may not improve outcomes in asymptomatic patients [7].

A multi-disciplinary approach (obstetricians, neonatologists, cardiologists and anaesthetists) with appropriate intraoperative monitoring and tailored anaesthetic technique is needed to achieve favourable outcomes. The use of continuous non-invasive cardiac output and blood pressure monitoring enabled timely intervention to maintain haemodynamic stability. The ease of use and rapid installation within minutes makes it suitable as a haemodynamic monitoring tool, especially in the urgent perioperative setting.

\section{References}

1. Martina JR, Westerhof BE, Van GJ, de Beaumont EM, Truijen J, et al. (2012) Noninvasive continuous arterial blood pressure monitoring with Nexfin. Anaesthesiology 116: 1092-1103. [Crossref]

2. Ganny AS, Eguma SA (2005) Intraoperative ventricular bigeminy: report of 5 cases. Annals of African Medicine 4: 72-82.

3. Sng BL, Wang H, Assam PN, Sia AT (2015) Assessment of an updated doublevasopressor automated system using Nexfin ${ }^{\mathrm{TM}}$ for the maintenance of haemodynamic stability to improve peri-operative outcome during spinal anaesthesia for caesarean section. Anaesthesia 70: 691-698. [Crossref]

4. Lawicka M, Malek A, Antczak D, Wajlonis A, Owczuk R (2015) Non-invasive haemodynamic measurements with Nexfin predict the risk of hypotension following spinal anaesthesia. Anaesthesiol Intensive Ther 47: 303-308.

5. Ameloot K, Vijver KVD, Broch O, Van Regenmortel N, De Laet I, et al. (2013) Nexfin Noninvasive Continuous Hemodynamic Monitoring: Validation against Continuous Pulse Contour and Intermittent Transpulmonary Thermodilution Derived Cardiac Output in Critically Ill Patients. ScientificWorldJournal article ID 519080. [Crossref]

6. Goldman L, Caldera DL, Nussbaum SR, Southwick FS, Krogstad D, et al. (1977) Multifactorial index of cardiac risk in noncardiac surgical procedures. $N$ Engl J Med 297: 845-850. [Crossref]

7. Siddoway LA (2003) Amiodarone: guidelines for use and monitoring. Am Fam Physician 68: 2189-2196. [Crossref]

Copyright: (C2017 Lim WY. This is an open-access article distributed under the terms of the Creative Commons Attribution License, which permits unrestricted use, distribution, and reproduction in any medium, provided the original author and source are credited. 